CLINICAL STUDY

\title{
Changes in expression of T-helper (Th) 1- and Th2-associated chemokine receptors on peripheral blood lymphocytes and plasma concentrations of their ligands, interferon-inducible protein-10 and thymus and activation-regulated chemokine, after antithyroid drug administration in hyperthyroid patients with Graves' disease
}

Yoshihisa Inukai, Atsushi Momobayashi ${ }^{1}$, Naoto Sugawara ${ }^{1}$ and Yoshimasa Aso

Department of Internal Medicine, Koshigaya Hospital, Dokkyo Medical University, 2-1-50 Minami-Koshigaya, Koshigaya, Saitama 343-8555, Japan and

${ }^{1}$ Mitsubishi Kagaku Bio-Clinical Laboratories, Inc., Tokyo 174-8555, Japan

(Correspondence should be addressed to Y Aso; Email: yaso@dokkyomed.ac.jp)

\begin{abstract}
Objective: Although Graves' disease is considered an autoantibody-mediated, T-helper 2 (Th2)dominant disease, Th1-dominance may prevail in its initial phase. We longitudinally investigated Th1/Th2 balance in untreated hyperthyroid patients with Graves' disease after treatment of methimazole (MMI), an antithyroid drug.

Design: University clinic outpatients were studied prospectively.

Patients: Subjects included 23 untreated hyperthyroid patients with Graves' disease and 17 agematched control subjects.

Methods: Before and after treatment, we measured Th1- and Th2-associated chemokine receptors (CXCR)3 and CCR4, on peripheral blood lymphocytes using flow cytometry, as well as plasma concentrations of their ligands, interferon-inducible protein (IP)-10 and thymus and activationregulated chemokine (TARC).

Results: The percentage of CXCR3-expressing cells among CD4 ${ }^{+} \mathrm{T}$ lymphocytes and plasma IP-10 was significantly higher in hyperthyroid Graves' disease patients than in controls. At 12 and 24 weeks after initiation of MMI, percentage of CXCR3-expressing CD4 ${ }^{+} \mathrm{T}$ lymphocytes had decreased significantly, while the percentage of CCR4-expressing CD4 ${ }^{+} \mathrm{T}$ lymphocytes had increased significantly at 24 weeks. The CXCR3/CCR 4 ratio had decreased significantly at 24 weeks. Plasma concentrations of IP-10 had decreased significantly at 12 and 24 weeks. Plasma concentrations of TARC also had decreased significantly at 24 weeks.

Conclusions: In hyperthyroid patients with Graves' disease in the active phase, Th1 cells rather than Th2 cells predominated among peripheral blood lymphocytes. After initiation of MMI, an ongoing transition from Th1 to Th2 dominance occurred.
\end{abstract}

European Journal of Endocrinology 156 623-630

\section{Introduction}

T-helper (Th) lymphocytes consist of two subpopulations, Th1 and Th2, based on distinctive patterns of cytokine production (1). Th1 lymphocytes, which secrete interferon (IFN)- $\gamma$ and interleukin (IL)-2, are associated strongly with cell-mediated immune responses, while Th2 lymphocytes secrete IL- 4 and IL5 and are involved in humoral immunity (2). Th2 immune responses also down-regulate Th1 immune responses (2). Since these subpopulations tend to function antagonistically toward one another, the balance between Th1 and Th2 lymphocytes may determine the outcome of autoimmune diseases (3).

Graves' disease, which causes hyperthyroidism, is an autoimmune disease involving the production of thyrotropin (TSH) receptor (TSHR) antibodies (TRAb), which overstimulate thyroid cells to result in hyperthyroidism (4). Graves' disease is characterized by persistent infiltration of the thyroid gland by lymphocytes composed mainly of CD4+ and CD8 + lymphocytes (4). A previous study demonstrated that thyroid glands affected by Graves' disease contained TSHR-specific T-cell clones that were characterized as Th2 on the basis of an 
increased ratio of IL-4 to IFN- $\gamma$ (5). Further, an in vitro study of cultured whole bloods from Graves' disease patients showed an increase in Th2-associated cytokine production (6). Graves' disease has long been thought to be an autoantibody-mediated, Th2-dominant autoimmune disease. However, using a model in which mice were injected with adenovirus expressing TSH receptor, Nagayama et al. demonstrated that an immune shift toward Th2 was accompanied by a decrease rather than an expected increase in production of thyroid stimulating antibody, which also suggested that predominant Th1 immune responses to TSH receptor are associated with induction of Graves' disease (7). More importantly, human TSH receptor stimulating antibodies (TSAbs) are predominantly immuno-globulin G (IgG)1, a Th1 type subclass in humans (8). Thus, the classification of Graves' disease as typical Th1 dominant disease is still under debate.

Chemokines are chemotactic cytokines controlling recruitment of leukocytes from the blood by regulating integrin adhesiveness (9). Based on structural motif specifically, the position of cysteine residues near their N-termini - chemokines have been divided into four subfamilies: a CXC family with one other amino acid between two cysteines; a CC family with no interposed amino acids; a C family with only one cysteine; and a CX3C family with three other amino acids between two cysteines (9). The chemokines act upon their effectors via specific receptors that belong to a seven-transmembrane-domain G protein-coupled family. The chemokine receptors CXCR3 and CCR 5 are expressed exclusively on Th1 lymphocytes (10), while Th2 lymphocytes preferentially express CCR4 and CCR3 (11). Recently, Romagnani et al. demonstrated recruitment of CXCR3-expressing Th1 lymphocytes and expression of the corresponding ligand, interferoninducible protein (IP)-10, in thyroid glands of patients at the initial phase of Graves' disease; this suggested Th1 rather than Th2 dominance within the thyroid gland at the onset of illness (12). Several other studies also reported increased serum IP-10 in patients with autoimmune thyroid diseases including Graves' disease (13-15). Thus, the assertion that Graves' disease is a Th2-driven disease is becoming increasingly controversial. A longitudinal study would be required to determine Th1/Th2 balance in patients with Graves' disease during the clinical course. However, no reports have described changes in Th1/Th2 balance in prospective analyses of chemokine receptor expression after treatment in these patients. Moreover, no reported studies have examined changes in plasma concentrations of ligands at these receptors, such as thymus and activation-regulated chemokine (TARC; a ligand for CCR4), after the treatment of Graves' disease.

We presently carried out a longitudinal investigation of Th1/Th2 balance in newly diagnosed hyperthyroid patients with Graves' disease, who were then given an antithyroid drug, by measuring Th1- and Th2asscoiated chemokine receptors on peripheral blood lymphocytes using flow cytometry as well as plasma concentrations of the receptor ligands.

\section{Materials and methods}

\section{Subjects}

We studied 23 untreated patients with Graves' disease (16 female and 7 male) and also 17 age-matched healthy control subjects. Subjects in the patient group had been referred to the outpatient clinic at the Dokkyo Medical University Hospital for treatment of hyperthyroidism. Patient age was $41.3 \pm 14.9$ years (21 to 63 ). Graves' disease was diagnosed based upon the presence of diffuse goiter, the elevated serum thyroid hormones (FT4 and FT3) and suppressed TSH, and the presence of TRAb. All patients with Graves' disease were treated with methimazole (MMI; 15-30 mg/day). The drug dose was decreased gradually whilst monitoring maintenance of normal thyroid function. At the end of the 24-week treatment period 21 were in a euthyroid state. Excluded from the study were patients with coexistent other organ-specific autoimmune disease; patients with medications that could affect the immune systems (such as corticosteroid) were also excluded, as were patients with allergic disease, such as those with atopic dermatitis or bronchial asthma. All patients gave informed consent. The study was approved by the local Ethics Committee of our institution.

\section{Methods}

Blood samples were obtained at study entry (baseline) after 12 weeks, and after 24 weeks of treatment. For plasma separation, venous blood was collected in a tube containing EDTA-2Na between 0800 and $0900 \mathrm{~h}$ after an overnight fast. After plasma samples were centrifuged at $2500 \boldsymbol{g}$ for $15 \mathrm{~min}$, the supernatant was stored at $-30{ }^{\circ} \mathrm{C}$ until use.

\section{Analysis of chemokine receptors by flow cytometry}

Heparinized whole blood $(0.1 \mathrm{ml})$ was incubated with antibodies to CXCR3 (DAKO Cytomation, Glostrup, Denmark), CCR4 phycoerythrin (Becton Dickinson, San Jose, CA, USA), and to CD4 peridinin chlorophyll protein (Becton Dickinson) conjugated all with carboxyfluorescein succinimidyl ester for $15 \mathrm{~min}$ at room temperature. Red blood cells were lysed by treating blood samples with ammonium chloride; white cells and then were resuspended in phosphate buffered saline, for analysis by a FACScan (Becton Dickinson). Receptor expression data were analyzed with CellQuest software (Becton Dickinson). Results are expressed as percentages of $\mathrm{CD} 4+$ cells expressing CXCR3 and CCR 4 , as well as the ratio of $\mathrm{CD} 4+$ cells bearing 
CXCR3 to those bearing CCR4. We regarded CXCR3 as a Th1-associated chemokine receptor, and CCR4 as a Th2-associated chemokine receptor.

\section{Measurements of chemokines in plasma}

Plasma concentrations of IP-10 were measured by ELISA (human IP-10 immunoassay, R\&D Systems, Minneapolis, MN, USA). Intra- and interassay coefficients of variation (CV) were $2.43-4.82 \%$ and $5.45-$ $8.26 \%$ respectively. Plasma concentrations of TARC also were measured by ELISA (human TARC immunoassay, R\&D Systems). Intra- and inter-assay CV were 2.90 $4.22 \%$ and $3.75-5.35 \%$ respectively.

TRAb was measured by a commercial RIA kit (TRAb CosmicIII, RSR, UK). Serum IgG was determined by an immunonephelometric assay (Dade Behring, Marburg, Germany). Serum IgE was measured by a fluoro-enzyme immunoassay (Phadia, Tokyo, Japan).

\section{Statistical analysis}

Data are expressed as the mean \pm s.D. or the median and interquartile ranges. Differences between groups were analyzed by a Student's paired $t$-test or an unpaired $t$-test. For nonparametric data, differences between groups were analyzed by Wilcoxon's matched paired test or the Mann-Whitney test. Correlation was determined by linear regression analysis. Logarithmic transformation of serum IgE concentration was used to render the distribution normal. A $P$ value below 0.05 was accepted as indicating statistical significance. Statistical analyses were carried out using SPSS 8.0 J software (SPSS, Tokyo, Japan).

\section{Results}

As shown in Table 1, both plasma IP-10 and TARC concentrations at the baseline were significantly higher in hyperthyroid patients with Graves' disease than in control subjects $(154.1 \pm 62.1$ vs $107.7 \pm 44.8 \mathrm{pg} / \mathrm{ml}$, $P=0.0078$; and $57.6 \pm 38.7$ vs $25.7 \pm 13.9 \mathrm{pg} / \mathrm{ml}, P=$ 0.0025 respectively). The percentage of CXCR 3 -expressing $\mathrm{CD} 4^{+} \mathrm{T}$ lymphocytes was significantly higher in hyperthyroid patients with Graves' disease than in control subjects $(27.0 \pm 7.0$ vs $22.6 \pm 5.5 \%, P=$ 0.0378 ), while we found no significant difference in CCR4-expressing cells among CD4 $+\mathrm{T}$ lymphocytes or in the CXCR3/CCR4 ratio between patients with hyperthyroid, patients with Graves' disease and control subjects (Table 1). At baseline, neither FT3 nor FT4 correlated with the percentage of CXCR3-expressing cells among $\mathrm{CD} 4+\mathrm{T}$ lymphocytes, the percentage of CCR4-expressing cells among $\mathrm{CD} 4+\mathrm{T}$ lymphocytes, and plasma concentrations of their ligands, IP-10 and TARC (data not shown). We also found no significant relationship between TRAb and the expression of
Table 1 Characteristics and laboratory data of control subjects and untreated patients with hyperthyroid Graves' disease.

\begin{tabular}{lccc}
\hline & Control & $\begin{array}{c}\text { Hyperthyroid } \\
\text { GD }\end{array}$ & $\boldsymbol{P}$ value \\
\hline$N$ (female/male) & $17(12 / 5)$ & $23(16 / 7)$ & \\
Age (years) & $37.1 \pm 12.8$ & $41.3 \pm 14.9$ & 0.3560 \\
Plasma IP-10 $(\mathrm{pg} / \mathrm{ml})$ & $107.7 \pm 44.8$ & $154.1 \pm 62.1$ & 0.0078 \\
Plasma TARC $(\mathrm{pg} / \mathrm{ml})$ & $25.7 \pm 13.9$ & $57.6 \pm 38.7$ & 0.0025 \\
CXCR3+/CD4+(\%) & $22.6 \pm 5.5$ & $27.0 \pm 7.0$ & 0.0378 \\
CCR4+/CD4+ $\%$ (\%) & $12.9 \pm 9.0$ & $12.2 \pm 5.4$ & 0.7503 \\
CXCR3+/CCR4+ ratio & $2.28 \pm 1.01$ & $2.78 \pm 1.70$ & 0.2878 \\
\hline
\end{tabular}

GD, Graves' disease; IP-10, interferon-inducible protein; TARC, thymus and activation-regulated chemokine. Data are mean \pm s.D.

chemokine receptors or their ligands concentration at baseline.

As shown in Table 2, both serum FT4 and FT3 were significantly reduced at 12 and 24 weeks after beginning MMI treatment. TRAb also was reduced at 12 and 24 weeks after treatment initiation. TRAb was lower at 12 weeks than at 24 weeks. Serum total IgG was significantly increased at 12 and 24 weeks after initiating treatment, while no significant changes in serum IgE were found.

Figure 1 displays representative flow cytometric results concerning changes in the percentage of CXCR3expressing or CCR4-expressing cells among peripheral CD4 + T lymphocytes after treatment with MMI in a typical patient with hyperthyroid Graves' disease. We found a marked reduction in the percentage of CXCR3expressing CD4 + Tlymphocytes and a marked increase in the percentage of CCR4-expressing $\mathrm{CD} 4+\mathrm{T}$ lymphocytes at 24 weeks compared with baseline.

After treatment with MMI, the percentage of CXCR3expressing CD4+ T lymphocytes decreased from 27.0土 $7.0 \%$ at baseline to $24.0 \pm 7.0 \%$ at 12 weeks and to $22.8 \pm 6.3 \%$ at 24 weeks $(P=0.0132$ and $P=0.0026$ respectively; Fig. 2A). Furthermore, the percentage of CXCR3-expressing cells was significantly lower at 24 weeks than at 12 weeks $(P=0.0426)$. On the other hand, the percentage of CCR4-expressing CD4 $+\mathrm{T}$ lymphocytes increased significantly, from $12.2 \pm 5.4 \%$ at baseline to $14.7 \pm 4.8 \%$ at 24 weeks $(P=0.0218)$ after beginning treatment (Fig. 2B). The percentage of CCR4-expressing cells was significantly higher at 24 weeks than at 12 weeks $(P=0.0147)$. The CXCR $3 / C C R 4$ ratio decreased significantly from $2.08(1.65,3.50)$ at baseline to 1.59 $(1.20,1.83)$ at 24 weeks $(P=0.0058)$ after beginning treatment (Fig. 3B). The ratio was also significantly lower at 24 weeks than at 12 weeks $(P=0.0288)$.

After initiation of MMI treatment, plasma concentrations of IP-10 decreased significantly, from $154.1 \pm 56.1$ at baseline to $91.3 \pm 34.1 \mathrm{pg} / \mathrm{ml}$ at 12 weeks and to $94.4 \pm 30.3 \mathrm{pg} / \mathrm{ml}$ at 24 weeks $(P=0.0002$ and $P=0.0001$ respectively; Fig. 4A). On the other hand, plasma concentrations of TARC decreased significantly, from $52.0(40.3,62.1)$ at baseline to $34.0(23.9,49.2)$ $\mathrm{pg} / \mathrm{ml}$ at 24 weeks $(P=0.0414$; Fig. $4 \mathrm{~B})$. 
Table 2 Changes in thyroid hormones, TRAb, total IgG, and IgE after treatment with methimazole in hyperthyroid patients with Graves' disease.

\begin{tabular}{lccc}
\hline & \multicolumn{3}{c}{ Duration of methimazole treatment } \\
\cline { 2 - 4 } & 0 week & 12 weeks & 24 weeks \\
\hline FT4 $(\mathrm{ng} / \mathrm{dl})$ & $5.25 \pm 1.90$ & $1.45 \pm 1.72^{\S}$ & $1.36 \pm 0.53^{\S}$ \\
FT3 $(\mathrm{pg} / \mathrm{ml})$ & $18.2 \pm 7.22$ & $6.23 \pm 5.66^{\S}$ & $4.20 \pm 1.47^{\S}$ \\
TSH $(\mu \mathrm{U} / \mathrm{ml})$ & $0.006(0.005,0.008)$ & $0.015(0.007,0.093)^{\star}$ & $0.034(0.012,1.038)^{\dagger}$ \\
TRAb $(\%)$ & $41.0(21.9,70.4)$ & $32.1(12.2,39.3)^{\star}$ & $18.6(10.1,41.4)^{\dagger}$ \\
Total lgG $(\mathrm{mg} / \mathrm{dl})$ & $1247 \pm 314.5$ & $1311 \pm 343.1^{*}$ & $1386 \pm 356.5^{\dagger}$ \\
lgE $(\log 10 \mathrm{mg} / \mathrm{dl})$ & $1.99 \pm 0.72$ & $2.03 \pm 0.71$ & $2.07 \pm 0.63$ \\
\hline
\end{tabular}

Data are mean \pm s.D. or median and interquartile. ${ }^{*} P<0.05,{ }^{\dagger} P<0.01,{ }^{\ddagger} P<0.001,{ }^{\S} P<0.0001$ vs 0 weeks, ${ }^{\natural} P<0.01$ vs 12 weeks. TRAb, thyrotropin (TSH) receptor antibodies; FT4, free thyroxine; FT3, free triiodothyronine. The reference ranges for laboratory values are: FT4, 0.97-1.79 ng/dl; FT3, 2.73$4.50 \mathrm{pg} / \mathrm{ml}$; TSH, $0.3-3.0 \mu \mathrm{U} / \mathrm{ml}$; TRAb, $0-15.0 \%$.

\section{Discussion}

The present study confirmed that plasma concentrations of IP-10, the ligand for CXCR3, were significantly higher in hyperthyroid patients with Graves' disease than in control subjects (13-16). Furthermore, plasma IP-10 decreased to concentrations resembling those in control subjects when a euthyroid state had been restored by MMI treatment. Antonelli et al. also reported that serum IP-10 was significantly higher in patients in the active phase of Graves' disease than in patients with Graves' disease rendered euthyroid by MMI treatment, suggesting that reduction in serum IP10 in patients treated with MMI may be associated with immunomodulatory effects of MMI $(14,15)$. They also demonstrated that circulating IP-10 was significantly higher in patients with Graves' disease than in those with toxic nodular goiter $(14,15)$, suggesting that hyperthyroidism itself is not associated with increased circulating IP-10. Thus, increased circulating IP-10 in patients with Graves' disease may be specifically sustained by the autoimmune, inflammatory process.
Immunohistochemical studies demonstrated high expression of IP-10 in thyroid glands of patients with recent-onset Graves' disease $(12,13,17)$. Thyroid follicular cells as well as infiltrating lymphocytes may be possible sources of IP-10 in sera from patients with newly diagnosed Graves' disease. Recently, Antonelli et al. have shown that in patients with Graves' disease, serum IP-10 is significantly reduced after thyroidectomy (18) or iodine-131 therapy (19). These results suggest that thyroid gland is the main source of circulating IP-10 in patients with Graves' disease. Increased plasma IP-10 would promote infiltration of activated lymphocytes into the thyroid gland, thus representing a potential indicator of disease activity in Graves' disease.

On the other hand, we found that plasma TRAC, a ligand for CCR 4, was significantly higher in hyperthyroid patients with Graves' disease than in control subjects. To our knowledge, this report is the first to demonstrate elevated plasma TARC concentrations in hyperthyroid patients with Graves' disease. This result supports the hypothesis that Th2-associated chemokines may
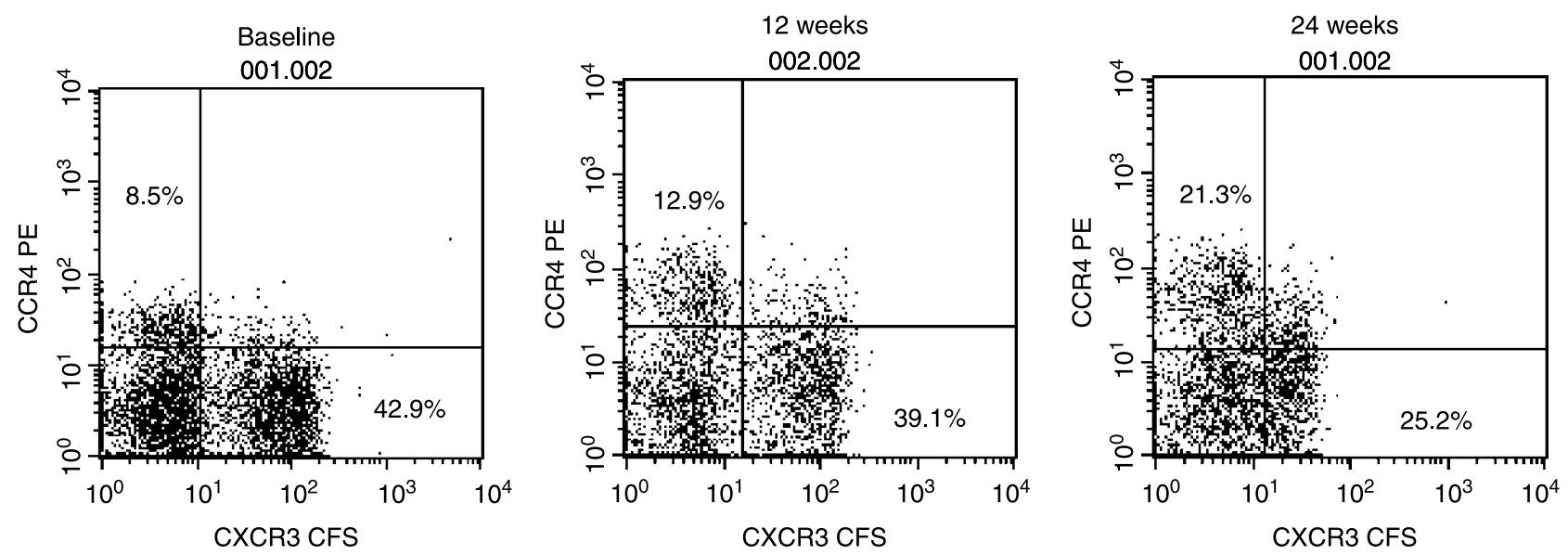

Figure 1 Representative results showing changes in the percentage of CXCR3-expressing or CCR4-expressing cells among CD4+ peripheral blood T lymphocytes after treatment with methimazole in a typical patient with hyperthyroid Graves' disease, as shown by flow cytometry. 

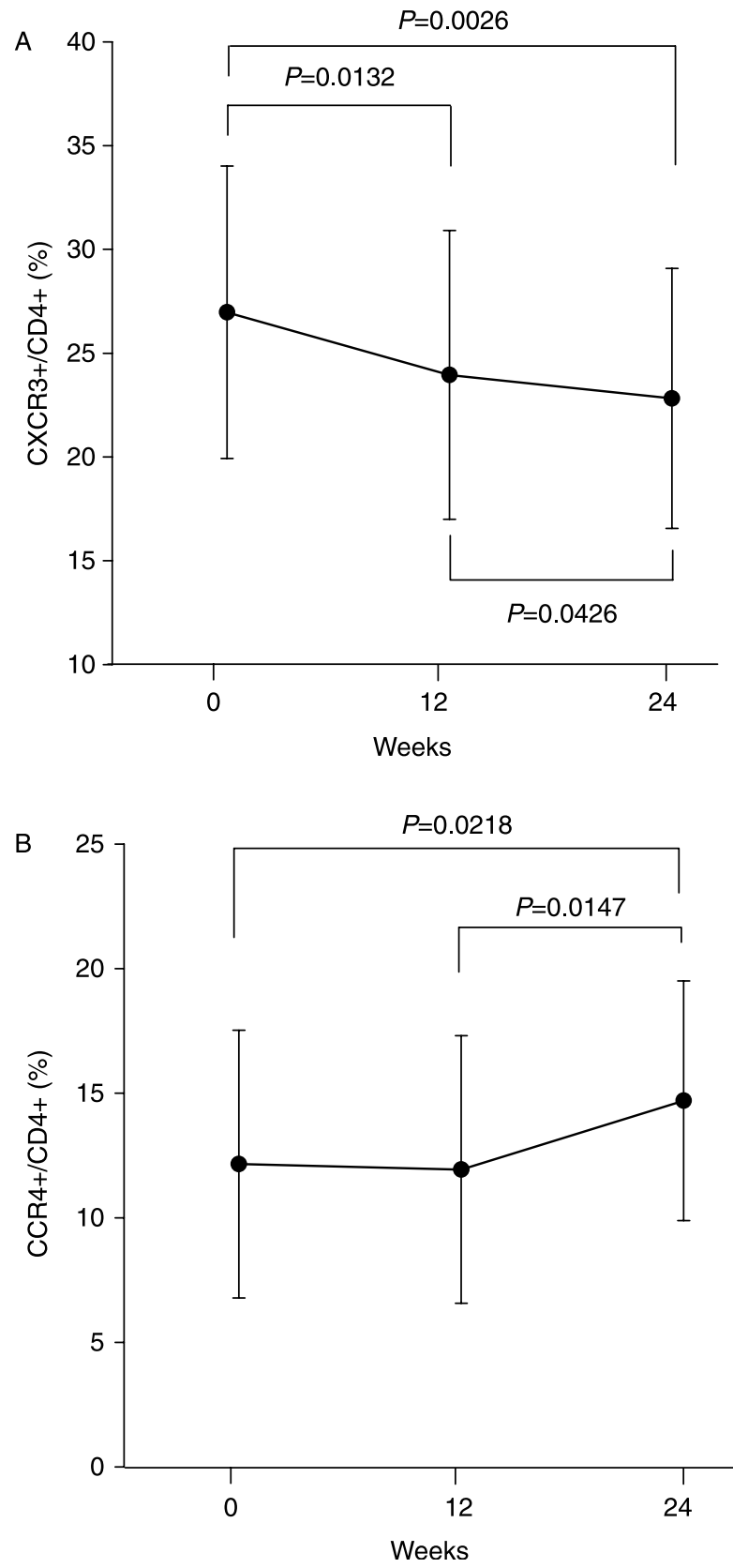

Figure 2 Changes in the percentage of CXCR3-expressing cells (A) or CCR4-expressing cells (B) among CD4 + peripheral blood T lymphocytes after treatment with methimazole in hyperthyroid patients with Graves' disease. Data are expressed as mean \pm s.D.

participate in the pathogenesis of Graves' disease. The present study also demonstrated that plasma TARC significantly decreased after treatment with MMI. Since a control group of toxic nodular goiter was not included in the present study, we cannot determine whether elevated plasma TARC concentrations are associated with the excess of thyroid hormones or the autoimmune inflammatory process in patients with Graves' disease.

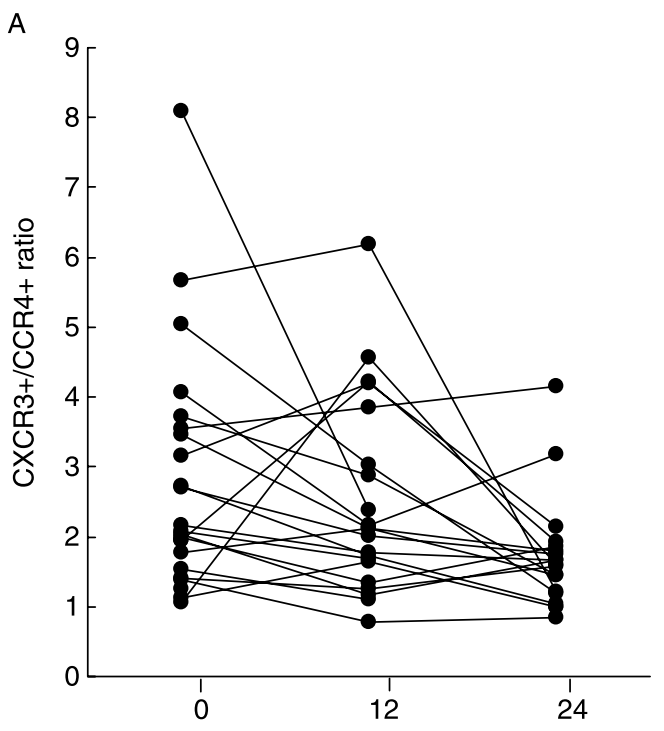

B

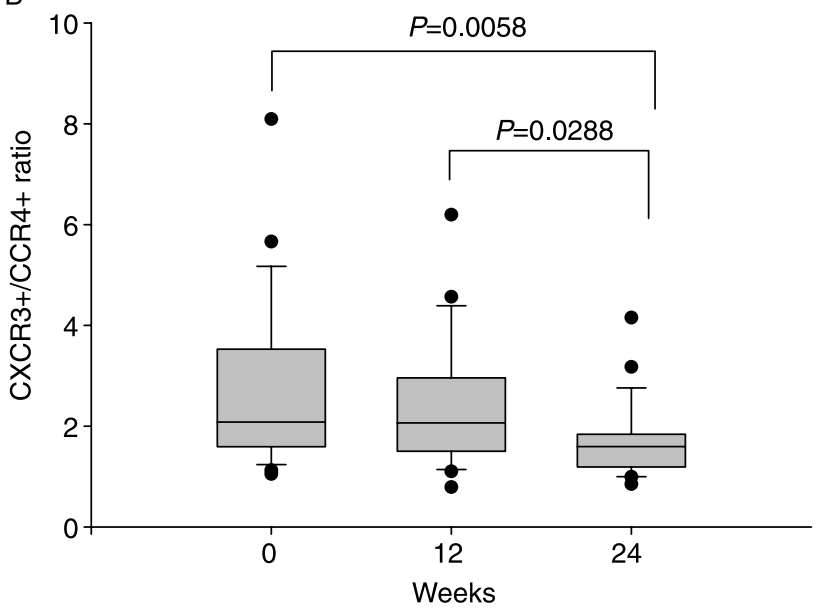

Figure 3 Changes in the CXCR3/CCR4 ratio after treatment with methimazole in hyperthyroid patients with Graves' disease. (A) Changes in the CXCR3/CCR4 ratio in individual patients. (B) Changes are expressed as median and interquartile. Boundaries of boxes, 25th and 75th percentiles; horizontal lines through boxes, median; whiskers, 10th and 90th percentiles; filled circles, outliers.

Similarly, a control group of toxic nodular goiter treated with MMI should be studied to investigate whether decreased plasma TARC after MMI is due to the transition from hyperthyroidism to euthyroidism or to the immunomodulatory effects of MMI. Several studies have reported that both plasma concentrations of TARC and expression of its receptor, CCR4, on peripheral blood CD $4+$ cells were increased in patients with atopic dermatitis, suggesting that elevated plasma TARC may reflect disease activity in atopic dermatitis (20-22). Furthermore, prevalence of CCR $4+$ cells among peripheral blood cells correlated positively with serum IgE in patients with atopic dermatitis (20), linking the pathogenesis of atopic dermatitis to Th2 dominance. 

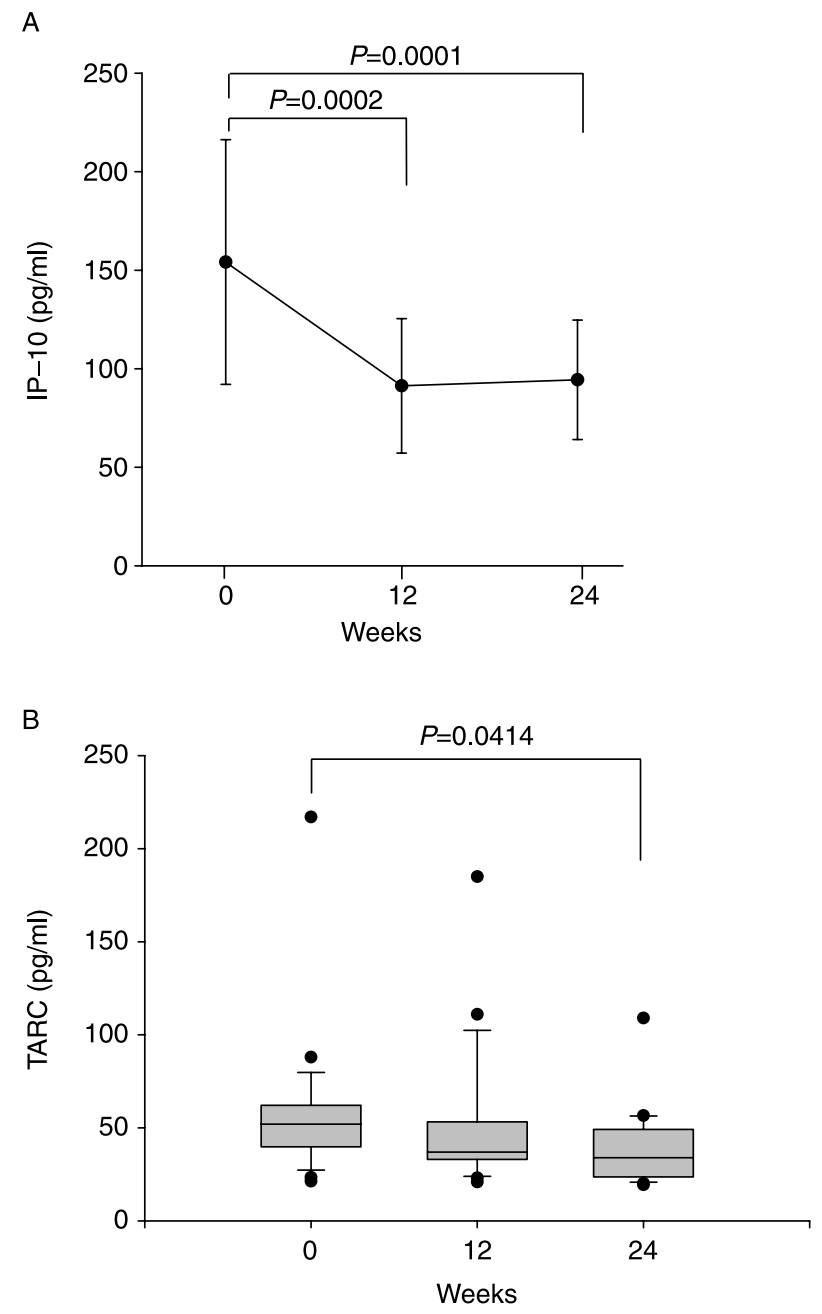

Figure 4 Changes in plasma concentrations of interferon-inducible protein (IP)-10 (panel A), and thymus and activation-regulated chemokine (TARC; panel B) in hyperthyroid patients with Graves' disease after treatment with methimazole. (A) Data are expressed as mean \pm s.D. (B) Data are expressed as median and interquartile. Boundaries of boxes, 25th and 75th percentiles; horizontal lines through boxes, median; whiskers, 10th and 90th percentiles; filled circles, outliers.

However, the present study showed the discordance between plasma concentrations of TARC and the percentage of CCR4-expressing cells among peripheral blood CD4 + cells in hyperthyroid patients with Graves' disease. One possible explanation for the discordance is that unlike atopic dermatitis, Th2 cells may not play a significant role in the active phase of Graves' disease. Another possibility involves the relatively small number of subjects studied, i.e., a type II error, which could be minimized by modestly increasing sample sizes.

We found a higher percentage of CXCR3-expressing cells among peripheral blood CD4 + cells in patients with hyperthyroid Graves' disease than in control subjects. This suggests that Th1 dominance may play an important role in disease induction. In essential disagreement, Aust et al. reported that the intrathyroidal lymphocytes from patients with Graves' disease showed higher expression of CXCR3 than autologous peripheral blood lymphocytes (23). No significant difference in percentage of CXCR3-postive cells was found between peripheral blood lymphocytes from their Graves' disease patients and those from their control subjects. However, their cross-sectional study compared the expression of CXCR3 on peripheral blood lymphocytes between Graves' disease patients in already rendered euthyroid after antithyroid drugs and control subjects (23). Thus, the discrepancy between the two studies may reflect a difference in timing of measurements with respect to treatment. In patients with Graves' ophthalmopathy, diffuse infiltration by lymphocytes is prominent in orbital fibroadipose tissues of Graves' as well as in the thyroid gland (24). Reflecting findings in the thyroid gland, a previous study in patients with recent-onset Graves' disease showed that cells cloned from the orbital tissues demonstrated a Th1 cell-mediated immune response predominating in the orbits, while Th2 immune response may predominate a later stage of disease such as after more than 2 years (24). A recent study has shown that serum concentrations of IP-10 are significantly higher in patients with active Graves' ophthalmopathy than in those with inactive Graves' ophthalmopathy, and that retrobulbar cells from patients with active Graves' ophthalmopathy can produce IP-10 under the influence of cytokine such as IFN- $\gamma$, while treatment of these cells with PPAR- $\gamma$ agonists suppress IFN- $\gamma$-induced IP-10 release (25).

Recruitment of lymphocytes in Graves' disease is a multistep process involving adhesion to and migration across the endothelium, movement through the interstitum, and finally contact with thyroid follicular cells (26). Chemokines and chemokine receptors play an important role in regulating traffic of effector $\mathrm{T}$ lymphocytes into inflamed areas (26). As mentioned previously, the ligand for CXCR3, IP-10, was also increased in plasma from patients with hyperthyroid Graves' disease. Thus, both high CXCR 3 expression and high plasma concentrations of the ligand IP-10 may contribute to migration of Th1 cells from peripheral blood to the affected thyroid gland in patients with hyperthyroid Graves' disease, which would result in accumulation of CXCR3-expressing cells in the thyroid gland.

The present study demonstrated for the first time that the percentage of CXCR3-expressing cells among peripheral blood CD4 + cells, representing Th1-associated lymphocytes, decreased significantly after MMI treatment in hyperthyroid patients with Graves' disease; on the other hand, the percentage of CCR4-expressing cells among peripheral blood CD $4+$ cells, representing Th2-associated lymphocytes, increased significantly after MMI treatment. In a cross-sectional study, Romagnani et al. immunohistochemically demonstrated 
high expression of CXCR3 and IP-10 proteins in mononuclear cells surrounding follicular structures in thyroid glands from patients with recent-onset Graves' disease, suggesting the importance of a Th1-dominant response in the initial phase of inflammation in this disease (12). However, no previous reports prospectively identified changes in Th1/Th2 balance according to expression of chemokine receptors and their ligands after the patients began treatment. This is the first study to longitudinally evaluate both the expression of chemokine receptors on peripheral blood lymphocytes and the corresponding ligands concentration in plasma after MMI treatment. We found that both the percentage of cells expressing CXCR 3 and the plasma concentrations of its ligand IP-10, which are Th1associated, were significantly decreased after MMI treatment; this suggested that the Th1/Th2 balance favored Th1 rather than Th2 dominance in the initial phase of Graves' disease. On the other hand, the present study showed a significantly increased percentage of CCR 4, a Th2-associated chemokine, after 24 weeks of treatment with MMI. The CXCR3/CCR4 ratio, indicating Th1/Th2 balance, also showed a significant decrease after MMI treatment compared with baseline. Furthermore, the ratio was significantly lower at 24 weeks of treatment than at 12 weeks. These results suggest that a progressive and gradual transition from Th1 dominance to Th2 dominance occurs during the clinical course of Graves' disease upon treatment with MMI, an antithyroid drug. Several studies also reported that peripheral lymphocytes reflect the autoimmunity in the thyroid gland as the expression of generalized activation of the immune system in patients with autoimmune thyroid disease (27-29). Thus, CXCR3expressing peripheral blood lymphocytes correlate with activity of Graves' disease, suggesting that the prevalence of such cells among peripheral blood lymphocytes would be a useful surrogate marker for thyroid autoimmune activity in this disease.

Whether or not MMI can directly induce a shift from Th1 dominance to Th2 dominance in the clinical course of Graves' disease remains unclear. In addition to lowering circulating thyroid hormones, MMI may have immunosuppressive effects (30). In fact, serum TRAb, a pathologic autoantibody, was found to decrease with time after administration of MMI (30). A previous study also showed decreased numbers of peripheral blood helper $\mathrm{T}$ lymphocytes and activated intrathyroidal T lymphocytes during antithyroid drug therapy (31). Thus, MMI is likely to directly decrease the expression of Th1-associated chemokine receptors and increase expression of Th2-associated chemokine receptors on peripheral blood lymphocytes, bringing about a change from Th1 dominance to Th2 dominance in the latter part of the clinical course. However, a control group treated with radioiodine or subtotal thyroidectomy should be studied to confirm a direct nature of MMI effects on the immune system in hyperthyroid patients with Graves' disease.

In conclusion, Th1 rather than Th2 cells were predominant among peripheral blood lymphocytes in hyperthyroid patients in the active phase of Graves' disease, suggesting that Th1 dominance is responsible for disease induction. After treatment with MMI, a progressive transition from Th1 to Th2 dominance was evidently found over time. Thus, CXCR3-expressing peripheral blood lymphocytes correlate with activity of Graves' disease, suggesting that the prevalence of such cells among peripheral blood lymphocytes would be a useful surrogate marker for thyroid autoimmune activity in this disease.

\section{Acknowledgements}

We wish to thank Professor T Inukai of our department for reviewing this manuscript and for his expert assistance, and Mr H Akabane as well as Mr S Takemoto of Mitsubishi Kagaku Bio-Clinical Laboratories (Tokyo, Japan) for their kind assistance. We also wish to thank our departmental colleagues for aiding us in this work.

\section{References}

1 Mosmann TR, Cherwinski H, Bond MW, Giedlin MA \& Coffman RL. Two types of murine helper T cell clone. I. Definition according to profiles of lymphokine activities and secreted proteins. Journal of Immunology $19861362348-2357$.

2 Mosmann TR \& Coffman RL. TH1 and TH2 cells: different patterns of lymphokine secretion lead to different functional properties. Annual Review of Immunology 19897 145-173.

3 Nicholson LB \& Kuchroo VK. Manipulation of the Th1/Th2 balance in autoimmune disease. Current Opinion in Immunology $19968837-842$.

4 Weetman AP. Graves' disease. New England Journal of Medicine $20003431236-1248$.

5 Mullins RJ, Cohen SB, Webb LM, Chernajovsky Y, Dayan CM, Londei $\mathrm{M} \&$ Feldmann M. Identification of thyroid stimulating hormone receptor-specific T cells in Graves' disease thyroid using autoantigen-transfected Epstein-Barr virus-transformed B cell lines. Journal of Clinical Investigation 1995 96 30-37.

6 Kallmann BA, Huther M, Tubes M, Feldkamp J, Bertrams J, Gries FA, Lampeter EF \& Kolb H. Systemic bias of cytokine production toward cell-mediated immune regulation in IDDM and toward humoral immunity in Graves' disease. Diabetes $1997 \mathbf{4 6}$ 237-243.

7 Nagayama Y, Mizuguchi H, Hayakawa T, Niwa M, McLachlan SM \& Rapoport B. Prevention of autoantibody-mediated Graves'-like hyperthyroidism in mice with IL-4, a Th2 cytokine. Journal of Immunology $2003 \mathbf{1 7 0} 3522-3527$.

8 McLachlan SM, Nagayama Y \& Rapoport B. Insight into Graves' hyperthyroidism from animal models. Endocrine Reviews 200526 800-832.

9 Luster AD. Chemokines-chemotactic cytokines that mediate inflammation. New England Journal of Medicine 1998338 436-445.

10 Qin S, Rottman JB, Myers P, Kassam N, Weinblatt M, Loetscher M, Koch AE, Moser B \& Mackay CR. The chemokine receptors CXCR3 
and CCR5 mark subsets of $\mathrm{T}$ cells associated with certain inflammatory reactions. Journal of Clinical Investigation 1998 $101746-754$.

11 Bonecchi R, Bianchi G, Bordignon PP, D’Ambrosio D, Lang R, Borsatti A, Sozzani S, Allavena P, Gray PA, Mantovani A \& Sinigaglia F. Differential expression of chemokine receptors and chemotactic responsiveness of type $1 \mathrm{~T}$ helper cells (Th1s) and Th2s. Journal of Experimental Medicine $1998 \mathbf{1 8 7} 129-134$.

12 Romagnani P, Rotondi M, Lazzeri E, Lasagni L, Francalanci M, Buonamano A, Milani S, Vitti P, Chiovato L, Tonacchera M, Bellastella A \& Serio M. Expression of IP-10/CXCL10 and MIG/CXCL9 in the thyroid and increased levels of IP-10/CXCL10 in the serum of patients with recent-onset Graves' disease. American Journal of Pathology 2002161 195-206.

13 Garcia-Lopez MA, Sancho D, Sanchez-Madrid F \& Marazuela M. Thyrocytes from autoimmune thyroid disorders produce the chemokines IP-10 and Mig and attract CXCR3 + lymphocytes. Journal of Clinical Endocrinology and Metabolism 200186 5008-5016.

14 Antonelli A, Fallahi P, Rotondi M, Ferrari SM, Romagnani P, Grosso M, Ferrannini E \& Serio M. Increased serum CXCL10 in Graves' disease or autoimmune thyroiditis is not associated with hyper- or hypothyroidism per se, but is specifically sustained by the autoimmune, inflammatory process. European Journal of Endocrinology $2006 \mathbf{1 5 4} 651-658$.

15 Antonelli A, Rotondi M, Fallahi P, Romagnani P, Ferrari SM, Barani L, Ferrannini E \& Serio M. Increase of interferon-gammainducible CXC chemokine CXCL10 serum levels in patients with active Graves' disease, and modulation by methimazole therapy. Clinical Endocrinology 200664 189-195.

16 Aso Y, Matsuura H, Momobayashi A, Inukai Y, Sugawara N, Nakano T, Yamamoto R, Wakabayashi S, Takebayashi K \& Inukai T. Profound reduction in T-helper (Th) 1 lymphocytes in peripheral blood from patients with concurrent type 1 diabetes and Graves' disease. Endocrine Journal 200653 377-385.

17 Kemp EH, Metcalfe RA, Smith KA, Woodroofe MN, Watson PF \& Weetman AP. Detection and localization of chemokine gene expression in autoimmune thyroid disease. Clinical Endocrinology $200359207-213$.

18 Antonelli A, Fallahi P, Rotondi M, Ferrari SM, Serio M \& Miccoli P. Serum levels of the interferon-gamma-inducible alpha chemokine CXCL10 in patients with active Graves' disease, and modulation by methimazole therapy and thyroidectomy. British Journal of Surgery $2006931226-1231$.

19 Antonelli A, Rotondi M, Fallahi P, Grosso M, Boni G, Ferrari SM, Romagnani P, Serio M, Mariani G \& Ferrannini E. Iodine-131 given for therapeutic purposes modulates differently interferon-gamma inducible alpha chemokine CXCL10 serum levels in patients with active Graves' disease or with toxic nodular goiter. Journal of Clinical Endocrinology and Metabolism 200792 1485-1490.
20 Sugawara N, Yamashita T, Ote Y, Miura M, Terada N \& Kurosawa M. TARC in allergic disease. Allergy 200257 180-181.

21 Leung TF, Wong CK, Chan IH, Ip WK, Lam CW \& Wong GW. Plasma concentration of thymus and activation-regulated chemokine is elevated in childhood asthma. Journal of Allergy and Clinical Immunology $2002110404-409$.

22 Nakatani T, Kaburagi Y, Shimada Y, Inaoki M, Takehara K, Mukaida N \& Sato S. CCR4 memory CD4+T lymphocytes are increased in peripheral blood and lesional skin from patients with atopic dermatitis. Journal of Allergy and Clinical Immunology 2001 107 353-358.

23 Aust G, Sittig D, Steinert M, Lamesch P \& Lohmann T. Graves' disease is associated with an altered CXCR 3 and CCR 5 expression in thyroid-derived compared to peripheral blood lymphocytes. Clinical and Experimental Immunology $2002127479-485$.

24 Aniszewski JP, Valyasevi RW \& Bahn RS. Relationship between disease duration and predominant orbital T cell subset in Graves' ophthalmopathy. Journal of Clinical Endocrinology and Metabolism $200085776-780$.

25 Antonelli A, Rotondi M, Ferrari SM, Fallahi P, Romagnani P, Franceschini SS, Serio M \& Ferrannini E. Interferon-gammainducible alpha-chemokine CXCL10 involvement in Graves' ophthalmopathy: modulation by peroxisome proliferator-activated receptor-gamma agonists. Journal of Clinical Endocrinology and Metabolism 200691 614-620.

26 Weetman AP. Cellular immune responses in autoimmune thyroid disease. Clinical Endocrinology 200461 405-413.

27 Gessl A, Wilfing A, Agis H, Steiner G, Czernin S, Boltz-Nitulescu G, Vierhapper H \& Waldhausl W. Activated naive CD4+ peripheral blood T cells in autoimmune thyroid disease. Thyroid $1995 \mathbf{5}$ $117-125$

28 Ciampolillo A, Guastamacchia E, Amati L, Magrone T, Munno I, Jirillo E, Triggiani V, Fallacara R \& Tafaro E. Modifications of the immune responsiveness in patients with autoimmune thyroiditis: evidence for a systemic immune alteration. Current Pharmaceutical Design 20039 1946-1950.

29 Mazziotti G, Sorvillo F, Naclerio C, Farzati A, Cioffi M, Perna R, Valentini G, Farzati B, Amato G \& Carella C. Type-1 response in peripheral CD4 + and CD8 + T cells from patients with Hashimoto's thyroiditis. European Journal of Endocrinology 2003148 383-388.

30 Cooper DS. Antithyroid drugs. New England Journal of Medicine 2005352 905-917.

31 Totterman TH, Karlsson FA, Bengtsson M \& Mendel-Hartvig I. Induction of circulating activated suppressor-like $\mathrm{T}$ cells by methimazole therapy for Graves' disease. New England Journal of Medicine 1987316 15-22.

Received 12 January 2007

Accepted 20 March 2007 